\title{
La langue et le style des écrivains. Claude Simon,
} L'Herbe

\section{Charles Camproux}

\section{(2) OpenEdition}

1 Journals

Édition électronique

URL : https://journals.openedition.org/ccs/605

DOI : $10.4000 /$ ccs.605

ISSN : 2558-782X

Éditeur :

Presses universitaires de Rennes, Association des lecteurs de Claude Simon

\section{Édition imprimée}

Date de publication : 30 novembre 2008

Pagination : 129-136

ISBN : 9782354120351

ISSN : 1774-9425

Référence électronique

Charles Camproux, "La langue et le style des écrivains. Claude Simon, L'Herbe », Cahiers Claude Simon [En ligne], 4 | 2008, mis en ligne le 21 septembre 2017, consulté le 27 avril 2022. URL : http:// journals.openedition.org/ccs/605; DOI : https://doi.org/10.4000/ccs.605 


\title{
La langue et le style des écrivains
}

\author{
Claude Simon, L'Herbe
}

par Charles CAMPROUX*

Toute question de lancement publicitaire mise de côté, je pense qu'il y a vraiment "quelque chose» dans ce que l'on est convenu d'appeler le « nouveau roman ». J'ignore s'il existe véritablement une « école» du nouveau roman : c'est affaire, d'ailleurs, aux critiques de l'histoire littéraire. Un chroniqueur de critique philologique («grammaire et philologie » suivant le terme officiel universitaire !) ne peut juger que sur pièces détaillées, et je m'excuse de ne point encore avoir pris connaissance de toute la production que, dès maintenant, on range sous la rubrique "nouveau roman». Mais il y a certainement une étroite parenté entre des ouvrages aussi différents cependant que «La modification » de Michel Butor, «La mise en scène » de Claude Oilier, et «L'herbe » de Claude Simon.

Et cette parenté réside indéniablement dans un style commun à ces romans, bien que différencié dans chacun d'eux. On retrouve dans "L'herbe » la hantise du temps et le mystère de sa nature (ce qui est, au fond, l'angoisse vitale), le sentiment de l'irréalité des choses réelles (ce qui, en fin de compte, nous donnera toujours la nausée contre quoi, d'instinct, l'homme essaie, tragiquement, de se révolter), et l'espèce de torture qui saisit l'être pensant en constadocument original à la BNF, à Anne-Lise Blanc pour la saisie informatique. 
tant le divorce entre pensée et conscience (ce qui légitime l'existence de l'écrivain et les tentatives de son œuvre). Et tout cela fait de «L'herbe » également un roman si l'on veut, mais un roman on ne peut plus antirécit. Le moindre paradoxe du nouveau roman, s'il continue à s'affirmer, ne sera pas d'avoir créé un genre qui sera aux antipodes du roman vrai. Mais on sait que le roman n'en est pas à un avatar près.

Les romanciers du nouveau roman semblent vouloir se défendre de toute attitude métaphysique, adopter une allure proprement scientifique, ne considérer leur objet que sous l'aspect d'une matière-là qu'il s'agit d'expliciter par une technique de l'écriture. L'entreprise peut être passionnante. Mais elle ne peut que se heurter à la matière « humaine ». Et d'abord à l'une des conditions essentielles de son existence même : la mouvance du temps. Ensuite à l'abîme, difficile, sinon impossible, à combler, qui sépare le sujet que, malgré tout, demeure le romancier, et la réalité qu'il entreprend d'expliciter ; et il ne sert à rien de déclarer que précisément cet abîme assure l'indépendance réciproque de l'auteur et de l'ouvrage à faire, si les tentatives de l'un ne peuvent s'achever dans les atteintes de l'autre. Enfin à l'impossibilité d'objectiver les faits de conscience en dehors de l'expression de la pensée.

À plusieurs reprises, l'auteur de «L'herbe », qui ne peut d'ailleurs pas s'opposer à sa lente mais continuelle poussée, essaie de maîtriser le temps mystérieux. Il compare les millésimes du carnet de raison de la vieille fille qui achève de mourir à «ces vestiges d'antiques constructions pélasgiques ou romaines et qui semblent non pas avoir résisté au temps mais être en quelque sorte le temps lui-même». Ailleurs, il écrit : "Cette période lui apparaîtra non comme une tranche de temps précise, mesurable et limitée, mais sous l'aspect d'une durée vague, hachurée, faite d'une succession, d'une alternance de trous, de sombres et de clairs ». Ou il évoque la libération " de la sujétion du présent»ou « une sorte de fabuleux mausolée fait de temps amoncelé sous un peu de cendre » et il rêve du " temps aboli ou resurgi ».

Claude Simon a su trouver un remarquable outil pour rendre sensible cette espèce de lutte titanesque engagée avec le temps qui est l'essentiel du roman : le participe présent. C'est, au vrai, un étour- 
dissant tour de force que d'avoir, en quelque sorte, écrit une longue phrase, ou presque, de 262 pages avec, à peu près comme seul temps et comme seul mode, le participe présent, sous sa forme propre de présent (que les linguistes appellent infectum) et sous sa variété de participe passé à valeur de présent parfait. Cet emploi du participe en -ant élimine absolument la notion de passé, de présent ou de futur. Pouvant s'employer pour circonstancier un autre verbe au passé, au présent ou au futur, si l'on supprime systématiquement tous les verbes à l'un de ces temps, par un artifice qui va naturellement contre toutes les habitudes de la langue organisée, il reste une sorte de ciment figé d'où est exclu tout mouvement temporel. Le procédé une fois admis, il faut bien reconnaître qu'on ne peut, sans doute, trouver mieux pour immobiliser le récit, les 262 pages du récit sousjacent au roman, en une sorte de fantasmagorie de Belle au Bois dormant. Il faut reconnaître que le procédé est excellemment bien choisi pour transformer des actions, des attitudes, des états d'âme qui n'ont pu réellement exister que dans la succession du temps, dans une espèce de mise en scène omniprésente qui ramène le tout à une plantation de choses-là : actes et sentiments.

Il est inutile de citer puisqu'il faudrait citer le roman tout entier. Mais il faut souligner que l'auteur n'hésite jamais à imbriquer ses participes les uns dans les autres, un participe se rapportant à un complément d'un participe précédent commandant à son tour un autre participe dont le complément se prolonge dans un nouveau participe qui à son tour...; le tout formant une sorte de glu immobilisant la réalité, personnages et leurs actes et leurs pensées s'interpénétrant, ce qui était ensuite devenant ce qui était auparavant, la glu s'étendant en tous sens et venant de tous les sens, une sorte de miroir non pas plan, mais spatial emprisonnant la vision non pas comprimée, mais s'échappant sans cesse, sans cesse revenant, finissant par étouffer l'attention critique, celle-ci, bercée, suivant sans plus d'opposition les suggestions d'une sorcellerie aboutissant à une méditation, cette méditation n'ayant d'ailleurs pas plus d'importance qu'une herbe croissant, croissant... Le grammairien n'a qu'à baisser les bras et à ne pas songer à essayer de rétablir l'analyse logique de telles pages où « la logique que nous avons pris l'habitude de voir régner dans les livres » n'a que peu à faire ; il acceptera de voir ce 
défilé de participes accaparant, escamotant plutôt, le temps, se chargeant de sujets différents et s'en déchargeant pour les recharger plus loin ; le participe prenant toutes les fonctions, aussi bien celle de proposition principale que de subordonnée en tout genre, puisque aussi bien dans cette mise en scène élargie jusqu'aux limites d'une absence d'horizon, tout est principal et rien n'est subordonné ou, si vous préférez, tout est subordonné et le principal est de ne pas savoir à quoi, tout en le devinant : c'est sans doute comme cela que les choses-là se présentent à la conscience. L'effet, en tout cas, est certain. Et réussi.

On s'aperçoit plus particulièrement de la valeur de ce participeroi dans les passages où, n'était l'artifice du principe adopté par le romancier, un récit vrai nous offrirait le passé simple dit de narration. "Louise sursauta, regarda la bossue... un peu plus tard, quand elle eut ouvert la boîte, elle resta là à regarder son contenu... » : ceci c'est du récit, c'est de la vie en mouvement racontée et donc de la vie successive, au petit détail ; la voulez-vous figer, l'immobiliser pour la réduire à l'état d'objets plantés là, pour essayer de la posséder tout à la fois afin de l'expliciter, de cette fusée toujours fuyante du temps, vous écrivez : "Louise sursautant (même si avant « Louise", il y a un point), regardant la bossue... un peu plus tard, quand elle eut ouvert la boîte, restant là à regarder son contenu... ».

Le passé simple dès lors devient disponible pour des tâches remarquables. Il permettra de souligner l'acte précis entre deux états, l'action brusque, rapide, ponctuelle; les instants où les choses se précipitent. En réalité, il est infiniment rare à travers «L'herbe» (qui ne croît pas par à-coups) : il ne s'agit pas d'un récit qui a besoin de progresser, ni d'une histoire qui veut être contée. Ce n'est nullement une progression dans le roman que marque l'emploi des rares passés simples que l'on y trouve, mais une sorte de convulsion interne de la réalité figée devant nos yeux ; comme une vague de fond, non pas une lame, une vague seulement, qui nous avertit que cette réalité vit intensément malgré l'apparente immobilité de la surface qui n'en paraît que davantage à nos sens comme un verre magique emprisonnant le mouvement, comme il est dit pour le miroir. Et l'on notera que ces quelques passés simples ne sont nullement des verbes principaux, des pivots de la phrase, mais qu'ils sont, tout au contraire, 
subordonnés à l'absence de mouvement général commandé par le participe, ligotés en quelque sorte par les multiples lianes sans cesse renaissantes projetées en tous sens par les participes - forêt vierge ; ainsi : "S'attendant à ce qu'il frappe, mais ne bougeant pas... ne le regardant pas, portant de temps en temps la cigarette à la bouche, regardant sortir la fumée, la fumée se détachant de ses lèvres, les paroles se détachant de ses lèvres, restant comme la fumée un instant suspendues..., une boule grise roulant sur elle-même, les sons prononcés, les mots roulant les uns sur les autres, c'est-à-dire montrant leurs diverses faces... puis (les mots, l'assemblage de mots) s'effilochant, se désagrégeant, se dissolvant dans l'air nocturne, mais cela ne vint pas (le coup, la gifle), Georges se tenant toujours immobile, elle pouvant voir les côtes maigres se soulevant et s'abaissant, et à la fin se décidant, elle, le visage parfaitement calme... laissant tout au plus... », etc.

Ce jeu remarquable du participe et du passé simple, s'il manifeste une sorte de tentative - que certains diront désespérée - de transmuer la nature du temps de façon que le temps lui-même devienne objet-là, révèle également un profond désir de comprendre la réalité au-delà de la déformation du langage organisé en langue. Il y a chez Claude Simon un sens aigu de la tragédie bien connue de tous les créateurs qui utilisent le langage comme outil de création ou de recréation : l'écrivain se débat contre la réalité qui le pénètre par tous les pores et qui tend à l'étouffer ; et son écriture n'est qu'une tentative pour surnager et dominer. Mais dès le moment où il surnage et domine, il ne pénètre plus : le réel lui échappe, il s'en sépare ; et là n'est pas la moindre difficulté des tentatives du nouveau roman qui voudrait précisément pénétrer et rendre compte de tout, en prenant ses distances avec tout considéré exclusivement en tant qu'objet. Cette contradiction, Claude Simon la souligne pertinemment quand il écrit : «Parce que le propre de la réalité est de nous paraître irréelle, incohérente du fait qu'elle se présente comme un perpétuel défi à la logique, au bon sens, du moins tels que nous avons pris l'habitude de les voir régner dans les livres - à cause de la façon dont sont ordonnés les mots, symboles graphiques ou sonores de choses, de sentiments, de passions désordonnées - si bien que naturellement il nous arrive parfois de nous demander laquelle de ces deux réalités 
est la vraie ». En écrivant ces mots, je ne suis pas loin de penser que Claude Simon a défini " l'art poétique » du nouveau roman, le nouveau romancier se donnant donc pour tâche de retrouver la réalité des choses désordonnées (par rapport à la langue organisée) malgré l'habitude prise dans les livres (et dans la grammaire, et dans les arts de rhétorique) d'ordonner les mots.

« L'herbe » illustre le précepte. De la première ligne à la dernière. Et non seulement l'emploi du verbe, mais tous les procédés de style qui concernent essentiellement la mise en ordre des mots. Le vocabulaire de «L'herbe » ne retient pas l'attention, pas plus que les comparaisons ou métaphores. Mais bien la syntaxe et la structure de la phrase. Les seules remarques qui peuvent concerner le vocabulaire portent précisément sur les mots les moins chargés d'image, les mots outils. Des mots comme « donc », « non pas », « mais », « pourtant», etc. n'ont point ici de valeur proprement logique : ils servent essentiellement d'accessoires pour essayer d'approcher la sensation, pour dénuder le réel. Caractéristique est l'emploi de "comme», l'outil ordinaire de la comparaison; dans "L'herbe», il est le plus souvent l'équivalent de "presque», n'introduit pas de comparaison et ne sert qu'à l'approche de la sensation. « Donc » n'a jamais sa valeur logique ; il sert presque toujours à résumer les longues parenthèses foisonnantes, les digressions envahissantes, à ramener l'attention sur la chose-là, immobile et vivante au milieu de la croissance immobile de l'herbe de la réalité-là. « Mais » n'est plus l'adversative des grammairiens, elle aussi ne sert plus qu'à contourner le réel pour mieux le voir, essayer de le saisir sous toutes ses faces afin d'exprimer l'incohérence insupportable de l'objet. C'est pourquoi tous ces mots outils, symboles du langage cohérent, perdent leur cohérence intrinsèque sous la plume de Claude Simon qui, conscient sans doute écrit : "C'est tout de même assez comique et même complètement absurde d'être obligé, de se croire obligé de s'exprimer d'une façon cohérente quand ce que l'on éprouve est incohérent. »

De là l'incohérence étudiée de la syntaxe. Souvent la construction grammaticale est oubliée. Une phrase, un membre de phrase plutôt (car il n'y a dans «L'herbe » que des membres de phrase et pas de vraies phrases) commençant par « non seulement en raison de 
leur prix » et suivi d'une longue subordonnée, est repris onze lignes plus loin par : «non seulement donc leur prix » : et « en raison de » disparaît dans l'épaisseur de l'herbe comme un puceron vert à peine entrevu dans son mouvement sans conséquence. Il va de soi que nombre de membres de phrase commençant par un participe en -ant restent en suspens et sont purement et simplement abandonnés. Si l'on essaie de distinguer une phrase de onze pages (entreprise difficile, puisque en général il n'y a pas de phrase), on se rendra compte que les temps formels du verbe y sont accumulés sans aucun lien grammatical logique. Un procédé soulignera davantage encore si possible cette asyntaxe : les paroles du style direct, lorsqu'il intervient, sont données à la queue leu leu sans ponctuation aucune, même pas entre les répliques; ce qui donne à six pages de style direct suivi l'apparence d'un bloc monolithe où le temps est nié, où la parole elle-même semble ne plus se dérouler mais être figée ; seule subsiste de-ci de-là la virgule passionnelle, simple ride de surface qui révèle malgré tout le rythme vital.

Il est certain que l'effet obtenu par de tels procédés, sacrilèges envers la syntaxe traditionnelle, vise et réussit souvent à " effacer toute pensée et toute formulation » pour ne laisser subsister que « la conscience » suivant la formule même de Claude Simon. C'est toute l'allure du style de "L'herbe ». De là l'abondance des parenthèses qui détruisent la formulation, qui de l'une à l'autre puis à l'autre encore ne laissent place qu'à une conscience vague qui ne pense plus mais subit le monde. De là les innombrables reprises, reprises correctives (non pas ceci, mais ainsi, ou plutôt ; non de... mais de ; ceci et même plus que ceci ; avec peut-être... ; et même pas ; etc.) ou reprises augmentatives qui donnent un mouvement imperceptible mais irrésistible au rythme ; de là les successions d'épithètes, de compléments introduisant d'énormes digressions, de précisions additionnelles, accumulatrices, sans plus aucun souci de la phrase bâtie, recherchant à l'opposé la phrase disloquée : «non pas donc de se débarrasser de, en finir avec, détruire ce qui dans la chambre... n'était déjà guère qu'une simple dépouille »: «chacun aux prises non plus avec une volonté adverse mais contre quelque chose en dehors de, échappant à toute volonté "; " feignant (allant au-devant de, prévenant, espérant détourner ainsi) la colère...»; etc. 
Il est évident que nous sommes avec "L'herbe" aux antipodes du récit bien conté, bien écrit. Est-ce encore du roman? En tout cas, c'est certainement une longue contemplation. Il y a bien une intrigue, puisque, tout comme dans «La modification» de Butor, le point d'arrivée nie le point de départ. Tout l'art de l'auteur est de placer le point d'arrivée sur le point de départ. Et de nous donner l'impression que nous ne sommes jamais partis. Tout est présent là, devant nous, même des successions d'années évoquées, comme on dit qu'au moment de la mort l'âme revoit tout son temps en un temps. Ce n'est plus le rythme ordinaire du roman, un rythme en déroulement. Ici, le rythme est vertical, en profondeur. Et c'est sans doute ce qui nous fascine. Nos nouveaux romanciers seraient-ils, en réalité, des poètes orientaux?

(C) Jean-Marie Petit 